\title{
Avaliação da rede de atenção primária do município de Recife - PE sob a ótica de um grupo de usuários
}

\author{
Evaluation of the Primary Care Network in the city of Recife-PE from the perspective of \\ a group of users
}

\author{
Carolina Gabriela Mota de Freitas ${ }^{1}$ \\ Orcid: https://orcid.org/0000-0002-0002-1966
}

\author{
Thaís Carine Lisboa da Silva ${ }^{2}$ \\ Orcid: https://orcid.org/0000-0001-9878-6280
}

\author{
Neciula de Paula Carneiro Porto Gomes ${ }^{3}$ \\ Orcid: https://orcid.org/0000-0001-9446-2553
}

\begin{abstract}
Resumo
Introdução: A Atenção Primária à Saúde tornou-se a principal porta de entrada e o primeiro contato do usuário com as redes de atenção à saúde, sendo de fundamental importância a sua efetivação nos municípios para a melhoria do sistema de saúde do Brasil. Nesse contexto, é imprescindível identificar a real aplicabilidade do modelo de serviço de saúde, juntamente com os seus impasses, tendo a percepção dos usuários como um importante instrumento de avaliação desses serviços. Objetivos/Métodos: Avaliar os atributos da atenção primária à saúde do Recife-PE sob a ótica dos usuários. Materiais e Métodos: Estudo observacional, analíticodescritivo, transversal. Teve como participantes 80 usuários adultos cadastrados nas Unidades de Saúde do Recife. Aplicou-se o instrumento Primary Care Assessment Tool - usuários adultos, versão reduzida. Resultados: Os componentes Grau de Afiliação, Acesso de Primeiro Contato -Utilização- e Coordenação -Integração do cuidado- atingiram escores médios superiores a 6,6, sendo bem avaliados. A dimensão Acesso de Primeiro Contato Acessibilidade- obteve escore inferior a 6,6, não atingindo a média estabelecida como desejável, comprometendo o alcance pleno do atributo Acesso de Primeiro Contato. Quanto à análise de relação entre a avaliação dos atributos e as variáveis sociodemográficas, apenas houve correlação entre a faixa etária e o acesso de primeiro contato -Utilização-. Conclusões: Embora os usuários reconheçam a Unidade de Saúde da Família como principal recurso de atenção à saúde e a valorizam como coordenadora do cuidado, ainda há dificuldades na acessibilidade ao serviço de saúde, necessitando de novas estratégias que direcionem maneiras de ampliação ao acesso.
\end{abstract}

Palavras-chave: atenção primária à saúde; avaliação de serviços de saúde; qualidade da assistência à saúde

\begin{abstract}
Introduction: Primary Health Care has become the main gateway and first contact the user has with the health care networks, being fundamental its accomplishment among cities to improve the Brazilian Health System. In this context, it is necessary to identify the real applicability of the health service model, along with its limitations, considering the perception of users as an important tool for evaluating these services. Objective: To evaluate the attributes of primary health care in Recife-PE from the perspective of users. Materials and Methods: Observational, analytical-descriptive, cross-sectional study. Participants were 80 adult registered users in the
\end{abstract}

\footnotetext{
${ }^{1}$ Faculdade Pernambucana de Saúde - FPS, Brasil. E-mail: carol.gmf@hotmail.com

${ }^{2}$ Faculdade Pernambucana de Saúde - FPS, Brasil. E-mail: thais_carine1@hotmail.com

${ }^{3}$ Faculdade Pernambucana de Saúde - FPS; IMIP; Faculdade Redentor-Recife, Brasil. E-mail: neciula@gmail.com
} 
Health Units of Recife. The Primary Care Assessment Tool - adult users, reduced version was applied. Results: The components Degree of Affiliation, First Contact Access - Utilizationand Coordination - Integration of care - reached average scores above 6.6, being well evaluated. The dimension First Contact Access - access to care - obtained scores below 6.6, not reaching the desirable established average, not allowing the full reach of the First Contact Access attribute. Regarding the relationship between the assessment of attributes and sociodemographic variables, there was only a correlation between the age group and the first contact access - utilization. Conclusions: Although users have been recognizing the Primary Care Health Centers as the main health care resource and value it as a care coordinator, still remain difficulties in the access to care into the health service, being necessary new strategies that make possible an increase in the access to the health care system.

Keywords: primary health care; health services research; quality of health care

\section{Introdução}

A partir da Conferência de AlmaAta, a Atenção Primária à Saúde (APS) pode ser compreendida como parte integrante e essencial dos sistemas de saúde, que tenham por objetivo garantir acesso universal, equânime, integral e sustentável à população assistida ${ }^{1}$.

No Brasil, o Programa de saúde da família (PSF), instituído em 1994, foi essencial para o fortalecimento da APS no país, ampliando o acesso aos cuidados à saúde, com ações que priorizam a promoção de saúde a partir do acompanhamento contínuo dos usuários, considerando o contexto familiar e comunitário, com equipes multiprofissionais atuando mais próximas da população e da comunidade. Em virtude dos excelentes resultados obtidos, após pouco mais de uma década de implantação, no ano de 2006, o PSF passou a ser reconhecido como a principal estratégia de reorganização da APS no Brasil, passando a se chamar de Estratégia de Saúde da Família (ESF) ${ }^{2}$.

Diante dessa conjuntura, foi publicada a Política Nacional de Atenção Básica $(\mathrm{PNAB})^{3}$ que, em sua última atualização, realizada em 2017, definiu a Atenção Básica (AB) como o "conjunto de ações de saúde individuais, familiares e coletivas que envolvem promoção, prevenção, proteção, diagnóstico, tratamento, reabilitação, redução de danos, cuidados paliativos e vigilância em saúde, desenvolvida por meio de práticas de cuidado integrado e gestão qualificada, realizada com equipe multiprofissional e dirigida à população em território definido, sobre as quais as equipes assumem responsabilidade sanitária" 3,4 .

Expoente nos estudos relacionados à APS, Bárbara Starfield ${ }^{5}$ sistematizou uma definição operacional da APS amplamente referenciada, inclusive internacionalmente, sendo também adotada pelo Ministério da Saúde no Brasil. A partir dessa, é possível identificar os atributos essenciais aos serviços de APS: o acesso de primeiro contato do indivíduo com o sistema de saúde, a longitudinalidade, a integralidade e a coordenação da atenção. Ademais, os três atributos derivados que qualificam as ações dos serviços de APS são: a atenção à saúde centrada na família (orientação familiar), orientação comunitária e competência cultural $^{5,6}$.

Um serviço de saúde que apresenta os quatro atributos essenciais pode ser considerado como um provedor de APS e, no caso de apresentar também os atributos derivados, tem o seu poder de interação com a família e a comunidade aumentado, potencializando, assim, os atributos essenciais ${ }^{5}$.

Há evidências sólidas, em diversas regiões do Brasil, da importância da APS para a manutenção da saúde da população, de acordo com diversos estudos que mostram uma forte relação da expansão da ESF com a redução de várias causas de mortalidade e morbidade no País ${ }^{7}$. Como evidenciado em pesquisas realizadas no nordeste brasileiro, que apontou um impacto significativo na diminuição dos 
casos de hanseníase em consonância com o aumento da cobertura dos serviços de $\mathrm{ESF}^{8}$ . Outro estudo realizado em um município da região sudeste, mostrou associação da redução da mortalidade infantil com o maior acesso às equipes de saúde da família $(\mathrm{ESF})^{9}$.

Apesar disso, como citado por Sousa e Hamann ${ }^{10}$, observam-se algumas dificuldades para a solidificação e expansão da ESF nos municípios, como dificuldade de acesso, a falta de articulação na rede assistencial e subfinanciamento. Além do mais, poucos estudos são direcionados a avaliar a efetividade e a qualidade da ESF de acordo com suas particularidades. Nesse caso, a percepção dos usuários é um excelente indicador para avaliação dos serviços de saúde, pois a perspectiva do usuário implica um julgamento sobre as características dos serviços e fornece informação essencial para completar e equilibrar a qualidade da atenção ${ }^{11,12}$

Em busca realizada nas bases bibliográficas de dados MEDLINE, via PubMed e SciELO, foram observados poucos estudos direcionados à avaliação dos serviços da APS segundo a visão dos usuários adultos no nordeste brasileiro. Tal fato pode ser evidenciado através da revisão sistemática realizada por Paula et al. ${ }^{13}$, em que foram incluídas pesquisas que utilizaram o instrumento PCATool para avaliar os atributos da APS de várias regiões do Brasil e que tiveram como população os usuários dos serviços. Este artigo mostrou que $87 \%$ das pesquisas selecionadas foram realizadas em municípios das regiões sul e sudeste do Brasil, ficando claro que os estudos da região Nordeste representam uma pequena parcela. Além disso, os artigos que se referem ao Nordeste, geralmente têm uma população amostral mais restritiva, como a pesquisa de Alves et $a l .{ }^{14}$, cujo objetivo foi avaliar a qualidade do cuidado na Atenção Primária à Saúde ofertado a usuários do sexo masculino segundo a sua perspectiva, realizada em um município da Paraíba.
Portanto, este estudo tem o objetivo de apresentar uma avaliação da presença do grau de afiliação e de dois atributos essenciais, o acesso de primeiro contato e a coordenação do cuidado, da rede de atenção primária do município do Recife em Pernambuco (PE) através da percepção dos usuários. Tal estudo torna-se relevante, pois abrange três aspectos que se entrelaçam, uma vez que somente uma APS fortalecida com um bom acesso ao serviço de saúde, e em uma boa afiliação, seria capaz de imputar a complexa missão de coordenar uma resposta integral em saúde.

\section{Materiais e Métodos}

\section{Amostra e tipo de estudo}

Trata-se de um estudo observacional analítico-descritivo de corte transversal, com abordagem quantitativa. $\mathrm{O}$ contexto sociodemográfico foi o município do Recife -PE e sua rede de APS que possui uma divisão administrativa em 8 distritos sanitários, do qual fazem parte 132 Unidades de Saúde da Família (USF) com população cadastrada e equipes multiprofissionais a elas vinculadas. As equipes de saúde da família cobrem cerca de 969.500 indivíduos, o que representa $59 \%{ }^{15}$ da população. Os participantes selecionados para essa pesquisa pertencem à população cadastrada nas unidades de saúde do Recife.

Para o presente estudo foi utilizada uma amostra probabilística de conveniência, portanto, para minimizar viéses na seleção dos indivíduos, foi realizado o sorteio aleatório de oito unidades de saúde, em que foram consideradas todas as USF's. De cada unidade selecionada, 10 usuários foram selecionados e entrevistados a esmo, formando a amostra da pesquisa com um total de 80 indivíduos.

O estudo foi aprovado pelo comitê de ética em pesquisa da Associação Educacional de Ciências da Saúde (AECISA) de Recife em 08/08/2019, sob 
Parecer $\quad \mathrm{n}^{\mathrm{o}}$ 3.494.949 (CAAE: 15856319.9.0000.5569).

\section{Delineamento da pesquisa}

A coleta de dados foi realizada nas unidades de saúde entre agosto e outubro de 2019. Foram 2 dias de visitas em cada unidade de saúde, com intervalos entre eles de 17 a 18 dias, a fim de abordar diferentes dias da semana, para que fossem entrevistados públicos de atividades diversificadas na unidade. Tal fato teve o objetivo de evitar viés de seleção, afastando a possibilidade de selecionar um perfil específico de usuário, como só gestantes ou participantes de programas de controle de doenças crônicas.

$\mathrm{Na}$ unidade de saúde foram entrevistados 5 usuários por dia, aleatoriamente, na sala de espera das USF, enquanto os mesmos aguardavam 0 atendimento. A duração da entrevista foi de aproximadamente 15 minutos.

\section{Critérios de Inclusão e Exclusão}

Como critério de inclusão, os entrevistados possuíam idade igual ou maior que 18 anos e eram usuários cadastrados em unidade de saúde do Recife que foram assistidos pelo serviço nos últimos 12 meses.

Foram excluídas deste estudo: pessoas menores de 18 anos, pessoas que nunca foram assistidas pelo serviço de atenção primária da RMR e pessoas que receberam atendimento nas unidades há mais de 12 meses.

\section{Procedimentos}

Para a avaliação dos serviços de saúde, existem vários instrumentos que podem ser aplicados, um deles foi criado por Starfield ${ }^{5}$ na Johns Hopkins Primary Care Policy Center, o PCATool (Primary Care Assessment Tool), um instrumento de Avaliação da Atenção Primária, apresentado originalmente em versões autoaplicáveis destinadas a crianças (PCATool versão Criança), a adultos maiores de 18 anos (PCATool versão Adulto) e a profissionais de saúde. Esse instrumento possui como alicerce o modelo de avaliação de qualidade dos serviços de saúde apresentado por Donabedian ${ }^{12}$, o qual considera a tríade "estrutura, processo e resultado", como sendo importantes informações sobre a qualidade dos cuidados.

Mesmo havendo outros instrumentos para avaliação da APS, o PCATool foi considerado o mais completo e adequado para mensurar os atributos necessários à APS, pois essa versão original mede a presença e a extensão dos atributos essenciais e atributos derivados da APS. Esse instrumento vem sendo muito utilizado no Brasil, inclusive é o instrumento recomendado pelo Ministério da Saúde ${ }^{6}$.

O PCATool, já validado em outros países, foi validado no Brasil e recebeu o nome de Instrumento de Avaliação da Atenção Primária - PCATool-Brasil, que se consolidou como um importante instrumento para avaliar serviços de saúde. A versão validada do PCATool do Adulto contém 87 itens, divididos em 10 componentes relacionados aos atributos da APS disponibilizado por meio do Manual do Instrumento de Avaliação da Atenção Primária à Saúde ${ }^{6,16}$.

Porém, vários autores já se utilizaram de adaptações reduzidas desse instrumento, inclusive no Brasil. Neste presente estudo, o instrumento foi usado em uma versão modificada, composto por onze questões que avaliaram a presença e extensão de dois atributos essenciais e suas respectivas dimensões: acesso de primeiro contato - utilização e acessibilidade; coordenação - integração do cuidado. Além disso, foi avaliado a dimensão grau de afiliação.

Os entrevistados foram convidados a responder às perguntas (Quadro 1), baseando-se na sua experiência da última 
consulta na rede de atenção primária do Recife-PE, nos últimos 12 meses anteriores à entrevista. Nas perguntas, os participantes tiveram que optar por uma das cinco respostas possíveis organizadas em uma escala Likert: "Certamente, sim" (pontuação = 4); "Provavelmente sim" (pontuação = 3); "Provavelmente não" (pontuação = 2); "Certamente não" (pontuação $=1$ ); e "Não sei ou não me lembro" (pontuação $=0$ ).

Quadro 1 - Questionário utilizado na entrevista, uma versão reduzida do instrumento Primary Care Assessment Tool (PCATool-Brasil) - usuários adultos.

\begin{tabular}{|c|c|}
\hline $\begin{array}{l}\text { Dimensões } \\
\text { avaliadas }\end{array}$ & Perguntas \\
\hline $\begin{array}{l}\text { Grau de } \\
\text { Afiliação }\end{array}$ & $\begin{array}{l}\text { Há um médico/enfermeiro ou serviço de saúde que você geralmente vai quando fica } \\
\text { doente ou precisa de conselhos sobre a sua saúde? }\end{array}$ \\
\hline \multirow{2}{*}{$\begin{array}{l}\text { Acesso de } \\
\text { primeiro } \\
\text { contato - } \\
\text { Utilização }\end{array}$} & $\begin{array}{l}\text { Quando você tem um novo problema de saúde, você vem à sua unidade de saúde antes } \\
\text { de ir a outro serviço de saúde? }\end{array}$ \\
\hline & $\begin{array}{l}\text { Quando você tem que consultar um especialista, o médico/enfermeiro da sua unidade de } \\
\text { saúde tem que encaminhar você, obrigatoriamente? }\end{array}$ \\
\hline \multirow{2}{*}{$\begin{array}{l}\text { Acesso de } \\
\text { primeiro } \\
\text { contato - } \\
\text { Acessibilidade }\end{array}$} & $\begin{array}{l}\text { É fácil marcar hora para uma consulta de revisão (consulta de rotina, “check-up”) na sua } \\
\text { unidade de saúde? }\end{array}$ \\
\hline & $\begin{array}{l}\text { Quando você chega na sua unidade de saúde, você tem que esperar mais de } 30 \text { minutos } \\
\text { para consultar com o médico ou enfermeiro (sem contar triagem ou acolhimento)? }\end{array}$ \\
\hline \multirow{6}{*}{$\begin{array}{l}\text { Coordenação - } \\
\text { Integração } \\
\text { do cuidado }\end{array}$} & $\begin{array}{l}\text { Você já foi consultar qualquer tipo de especialista ou serviço especializado no período } \\
\text { em que você está em acompanhamento na sua unidade de saúde? }\end{array}$ \\
\hline & $\begin{array}{l}\text { O seu médico/enfermeiro sugeriu (indicou, encaminhou) que você fosse consultar com } \\
\text { esse especialista ou serviço especializado? }\end{array}$ \\
\hline & $\begin{array}{l}\text { O seu médico/enfermeiro discutiu com você diferentes serviços em que você pudesse } \\
\text { ser atendido para esse problema de saúde? }\end{array}$ \\
\hline & $\begin{array}{l}\text { O seu médico/enfermeiro escreveu alguma informação para o especialista, a respeito do } \\
\text { motivo dessa consulta? }\end{array}$ \\
\hline & O médico/ enfermeiro sabe quais foram os resultados dessa consulta? \\
\hline & $\begin{array}{l}\text { O seu médico/enfermeiro pareceu interessado na qualidade do cuidado que lhe foi dado } \\
\text { (lhe perguntou se você foi bem ou mal atendido por esse especialista ou serviço } \\
\text { especializado)? }\end{array}$ \\
\hline
\end{tabular}

Os dados coletados passaram por uma revisão dos questionários para verificar a legibilidade e a qualidade da informação coletada. Após isso, os questionários foram agrupados conforme as unidades de saúde de cada região e distrito. Para digitação dos dados, foi utilizado o software Microsoft
Excel® 2019 e realizado dupla digitação, em épocas e por pessoas diferentes, que compararam posteriormente os dois bancos e corrigiram eventuais erros ou inconsistências. Somente depois de comparados os bancos é que o banco de dados definitivo foi utilizado para análise 
estatística, no software Statistical Package for the Social Sciences (SPSS), versão 21, no qual se realizou tanto a análise descritiva das variáveis quanto o teste qui-quadrado de Pearson para verificar associação entre as variáveis qualitativas. $\mathrm{O}$ nível de significância adotado para os testes foi de $5 \%$ e o intervalo de confiança, de $95 \%$.
O cálculo do escore de desempenho da APS seguiu o manual do PCAToolBrasil $^{6}$ do Ministério da Saúde. Por isso, foi necessário, primeiramente, observar que, na escala original do instrumento, as respostas do tipo likert possuem cada alternativa um valor, conforme quadro abaixo:

Quadro 2 - Valores das respostas das questões 2,3,4,7,8,9,10 e $11^{*+*}$

\begin{tabular}{|c|c|c|c|c|c|}
\hline $\begin{array}{c}\text { Respostas } \\
\text { possíveis }\end{array}$ & $\begin{array}{c}\text { Com } \\
\text { certeza, } \\
\operatorname{sim}\end{array}$ & $\begin{array}{c}\text { Provavelmente, } \\
\operatorname{sim}\end{array}$ & $\begin{array}{c}\text { Provavelmente, } \\
\text { não }\end{array}$ & $\begin{array}{c}\text { Com } \\
\text { certeza } \\
\text { não }\end{array}$ & $\begin{array}{c}\text { Não } \\
\text { sei/Não } \\
\text { lembro }\end{array}$ \\
\hline $\begin{array}{c}\text { Valor } \\
\text { Correspondente }\end{array}$ & 4 & 3 & 2 & 1 & 9 \\
\hline
\end{tabular}

*O questionário de número 1 teve valor $=1$ quando a resposta foi NÃO, e valor $=4$ quando a resposta foi SIM. ${ }^{\dagger} \mathrm{O}$ questionário de número 5 foi formulado de maneira que quanto maior o valor (resposta) atribuído, menor é a orientação para APS. Logo, esses itens tiveram seus valores invertidos para: (valor 4=1), (valor 3=2), (valor 2=3), (valor $1=4)$ ) ${ }^{\star} \mathrm{O}$ questionário de número 6 não teve valor avaliativo. Foi utilizado apenas para dar seguimento as outras questões.

Foi calculado o escore da APS para cada uma das dimensões analisadas. Cada componente foi avaliado pela média aritmética dos questionários correspondentes, ou seja:

1. Grau de Afiliação (questão1):

Foi avaliado apenas pela pergunta de número 1, portanto, não foi necessário calcular a média aritmética. Nesse caso, os possíveis escores foram 1 ou 4 para esse atributo.

2. Acesso de primeiro contato utilização (questões 2 e 3 ):

$\mathrm{O}$ escore para esse componente foi calculado pela soma do valor dos itens dividido por 2, ou seja, Escore $=\mathrm{Q} 2+\mathrm{Q} 3 / 2$.

3. Acesso de primeiro contato acessibilidade (questões 4 e $5^{*}$ ):

*A questão 5 tem o valor invertido, portanto, foi calculado esse componente após ser convertido pelo valor correspondente.

O escore para esse componente foi calculado pela soma do valor dos itens dividido por 2, ou seja, Escore $=$ Q4+Q5/2.
4. Coordenação - integração do cuidado (questões* 7, 8, 9, 10 e 11): *A questão 6 não entrou no cálculo do escore por se tratar de um item descritivo.

$\mathrm{O}$ escore para esse componente foi calculado pela soma do valor dos itens dividido por 5, ou seja, Escore = Q7+Q8+Q9+Q10+Q11/5.

Em um segundo momento, todas as pontuações, para cada um dos atributos, foram posteriormente transformadas em uma escala que varia de 0 (zero) a 10 (dez) pontos, de acordo com o instrumento de validação do PCATool-Brasil em sua versão para adultos. A padronização para a escala $0-10$ foi feita da seguinte forma: Escore padronizado $=[($ Escore obtido -1$)$ $\mathrm{x} 10] / 3$.

Para a conversão das escalas, foram observadas essas duas seguintes situações:

1. Se para um entrevistado, a soma de respostas em branco com respostas "9” ("não sei/não lembro") atingiu $50 \%$ ou mais do total de itens de um atributo analisado, não foi calculado 
o escore desse componente para esse entrevistado. O escore desse componente para esse entrevistado ficou em branco no banco de dados.

2. Se para um entrevistado, a soma de respostas em branco com respostas "9" ("não sei/não lembro") foi inferior a $50 \%$ do total de itens de um atributo analisado, foi realizada a transformação do valor "9" para o valor "2" ("provavelmente não"). Essa transformação foi necessária para pontuar negativamente algumas características do serviço de saúde que não são conhecidas pelo entrevistado.

Para análise dos escores, os valores iguais ou superiores a 6,6 foram considerados de alto escore, pois correspondem a respostas para as opções 3 (provavelmente, sim) ou 4 (com certeza, sim) na escala original do instrumento. Os resultados foram apresentados por meio de descrições das frequências relativas $\mathrm{e}$ absolutas.

\section{Resultados}

Foram entrevistados 80 usuários, vinculados a 8 USF do Recife, em Pernambuco. Quanto ao perfil dos entrevistados, notou-se que a maioria era do sexo feminino $(82,50 \%)$, adultos entre 25 e 59 anos $(52,50 \%)$, com renda familiar de até 1 salário mínimo $(63,75 \%)$ e com composição familiar de 4 pessoas ou mais $(42,50 \%) .68,75 \%$ se autodeclararam pretos ou pardos e $57,50 \%$ possuíam 10 anos de estudo ou mais (Tabela 1).

Tabela 1 - Perfil sociodemográfico dos usuários da atenção primária em Recife, PE, Brasil, 2019.

\begin{tabular}{|c|c|c|c|c|c|}
\hline & \multirow{2}{*}{ Variáveis } & \multicolumn{4}{|c|}{ Usuários APS ( $N=80 *)$} \\
\hline & & \multirow{2}{*}{$\begin{array}{l}\mathbf{n} \\
66\end{array}$} & \multirow{2}{*}{$\begin{array}{c}\% \\
82,50 \%\end{array}$} & \multicolumn{2}{|c|}{ IC $95 \% \dagger$} \\
\hline \multirow{2}{*}{ Sexo } & Feminino & & & $73,10 \%$ & $89,60 \%$ \\
\hline & Masculino & 14 & $17,50 \%$ & $10,40 \%$ & $26,90 \%$ \\
\hline \multirow{3}{*}{ Idade } & Entre 18 e 24 anos & 16 & $20,00 \%$ & $12,39 \%$ & $29,74 \%$ \\
\hline & Entre 25 e 59 anos & 42 & $52,50 \%$ & $41,63 \%$ & $63,19 \%$ \\
\hline & 60 anos ou mais & 22 & $27,50 \%$ & $18,64 \%$ & $37,96 \%$ \\
\hline \multirow{3}{*}{ Cor/Raça } & Brancos & 15 & $18,75 \%$ & $11,39 \%$ & $28,33 \%$ \\
\hline & Pretos e Pardos & 55 & $68,75 \%$ & $58,07 \%$ & $78,10 \%$ \\
\hline & Outros & 10 & $12,50 \%$ & $6,61 \%$ & $21,04 \%$ \\
\hline \multirow{3}{*}{$\begin{array}{l}\text { Renda } \\
\text { Familiar }\end{array}$} & Até 1 salário mínimo & 51 & $63,75 \%$ & $52,88 \%$ & $73,65 \%$ \\
\hline & De 1 a 2 salários mínimos & 25 & $31,25 \%$ & $21,90 \%$ & $41,93 \%$ \\
\hline & Mais do que 2 salários mínimos & 4 & $5,00 \%$ & $1,71 \%$ & $11,45 \%$ \\
\hline \multirow{3}{*}{$\begin{array}{l}\text { Composição } \\
\text { Familiar }\end{array}$} & Até 2 pessoas & 22 & $27,50 \%$ & $18,64 \%$ & $37,96 \%$ \\
\hline & 3 pessoas & 24 & $30,00 \%$ & $20,80 \%$ & $40,62 \%$ \\
\hline & 4 pessoas ou mais & 34 & $42,50 \%$ & $32,09 \%$ & $53,44 \%$ \\
\hline \multirow{3}{*}{ Escolaridade } & Sem escolaridade & 5 & $6,25 \%$ & $2,42 \%$ & $13,15 \%$ \\
\hline & Até 9 anos de estudo & 29 & $36,25 \%$ & $26,35 \%$ & $47,12 \%$ \\
\hline & 10 anos de estudo ou mais & 46 & $57,50 \%$ & $46,56 \%$ & $67,91 \%$ \\
\hline
\end{tabular}

Legenda: APS - Atenção Primária à saúde; ${ }^{*} \mathrm{~N}=80$ corresponde à população total da amostra; ${ }^{\dagger} \mathrm{IC} 95 \%$ - Intervalo de $95 \%$ de confiança; ${ }^{*}$ Salário-mínimo vigente no período da pesquisa era de R\$ 998,00. 
A Tabela 2 apresenta a média dos escores gerais e as respectivas porcentagens do alto e do baixo escore de cada dimensão: grau de afiliação, acesso de primeiro contato - utilização, acesso de primeiro contato - acessibilidade e coordenação integração do cuidado. Constatou-se que o
Grau de Afiliação, acesso de primeiro contato - utilização e coordenação integração do cuidado atingiram escore médio superior a 6,6 , sendo assim bem avaliados. $O$ componente acesso de primeiro contato - acessibilidade, obteve escore inferior a 6,6 .

Tabela 2 - Valores médios das dimensões e os respectivos percentuais de avaliação de alto e de baixo escore de cada dimensão avaliada.

\begin{tabular}{|c|c|c|c|c|}
\hline & $\begin{array}{l}\text { Valor Médio* } \\
\qquad(\text { 0-10 ) }\end{array}$ & $\begin{array}{l}\text { Alto Escore } \\
\quad(\geq 6,6)\end{array}$ & $\begin{array}{l}\text { Baixo Escore } \\
(<6,6)\end{array}$ & $\begin{array}{c}\text { Não } \\
\text { Avaliado }\end{array}$ \\
\hline & $\begin{array}{c}\text { Média } \\
\left(\text { IC } 95 \%{ }^{\dagger}\right)\end{array}$ & $\begin{array}{c}\mathrm{n} \\
(\%)\end{array}$ & $\begin{array}{c}\mathrm{n} \\
(\%)\end{array}$ & $\begin{array}{c}\mathrm{n} \\
(\%)\end{array}$ \\
\hline Grau de Afiliação & $\begin{array}{c}8,9 \\
(8,2-9,6)\end{array}$ & $\begin{array}{c}71 \\
(88,75 \%)\end{array}$ & $\begin{array}{c}9 \\
(11,25 \%)\end{array}$ & - \\
\hline $\begin{array}{c}\text { Acesso de primeiro contato } \\
\text { - Utilização }\end{array}$ & $\begin{array}{c}7,7 \\
(7,2-8,2)\end{array}$ & $\begin{array}{c}63 \\
(78,75 \%)\end{array}$ & $\begin{array}{c}17 \\
(21,25 \%)\end{array}$ & - \\
\hline $\begin{array}{l}\text { Acesso de primeiro contato } \\
\text { - Acessibilidade }\end{array}$ & $\begin{array}{c}3,3 \\
(2,7-3,9)\end{array}$ & $\begin{array}{c}11 \\
(13,75 \%)\end{array}$ & $\begin{array}{c}69 \\
(86,25 \%)\end{array}$ & - \\
\hline $\begin{array}{c}\text { Coordenação } \\
\text { - Integração do cuidado }\end{array}$ & $\begin{array}{c}6,8 \\
(6,2-7,3)\end{array}$ & $\begin{array}{c}36 \\
(45,00 \%)\end{array}$ & $\begin{array}{c}24 \\
(30,00 \%)\end{array}$ & $\begin{array}{c}20 \\
(25,00 \%)\end{array}$ \\
\hline
\end{tabular}

Legenda: * Corresponde à média aritmética dos escores da APS avaliados de acordo com cada uma das dimensões, numa escala de $0-10 ;{ }^{\dagger}$ IC $95 \%$ : Intervalo de $95 \%$ de confiança

As Tabelas 3, 4, 5 e 6 detalham a variabilidade da avaliação das dimensões em relação aos fatores sociodemográficos contemplados neste estudo. Ao analisar cada dimensão separadamente, identificouse que apenas um componente do atributo "acesso de primeiro contato" (dimensão "utilização") se relacionou com um dos fatores considerados. Em valores percentuais, a avaliação dos usuários com
60 anos ou mais se associou com um melhor "acesso de primeiro contato - utilização", com diferença estatisticamente significativa $(\mathrm{p}=0,036)$ (tabela 4).

As demais dimensões (grau de afiliação, acesso de primeiro contatoacessibilidade e coordenação - integração dos cuidados), conforme tabelas 3,5 e 6 , foram avaliadas de forma homogênea em relação às variáveis consideradas $(p>0,05)$.

Tabela 3 - Associação do componente Grau de Afiliação com as variáveis sociodemográficas.

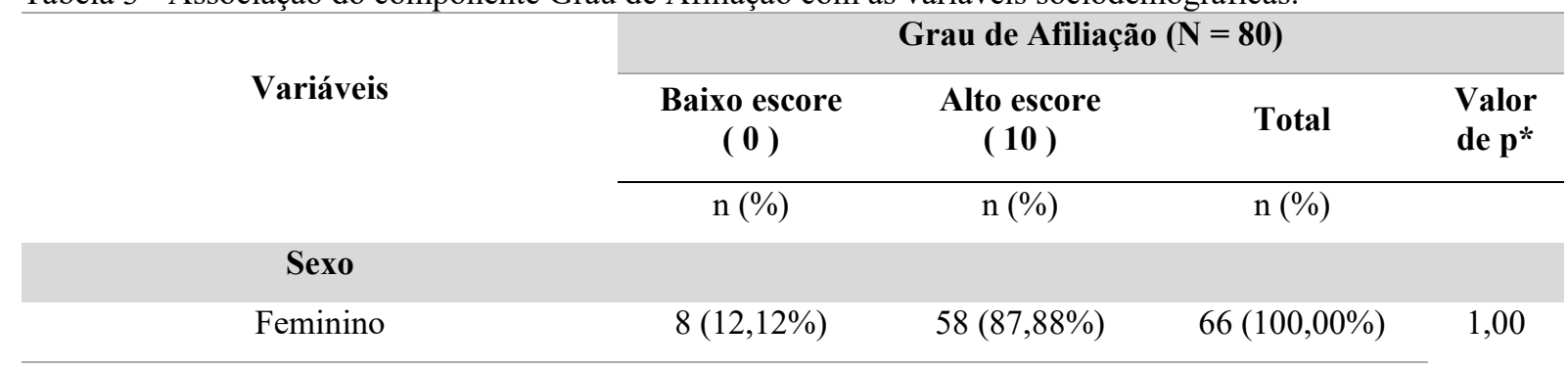


Avaliação da Atenção Primária à Saúde

Evaluation of the Primary Health Care

Masculino $\quad 1(7,14 \%) \quad 13(92,86 \%) \quad 14(100,00 \%)$

\begin{tabular}{|c|c|c|c|c|}
\hline Idade & & & & \\
\hline Entre 18 e 24 anos & $0(0,00 \%)$ & $16(100,00 \%)$ & $16(100,00 \%)$ & \multirow{3}{*}{0,249} \\
\hline Entre 25 e 59 anos & $7(16,67 \%)$ & $35(83,33 \%)$ & $42(100,00 \%)$ & \\
\hline 60 anos ou mais & $2(9,09 \%)$ & $20(90,91 \%)$ & $22(100,00 \%)$ & \\
\hline \multicolumn{5}{|l|}{ Cor/Raça } \\
\hline Brancos & $1(6,67 \%)$ & $14(93,33 \%)$ & $15(100,00 \%)$ & \multirow{3}{*}{0,646} \\
\hline Pretos e Pardos & $6(10,91 \%)$ & $49(89,09 \%)$ & $55(100,00 \%)$ & \\
\hline Outros & $2(20,00 \%)$ & $8(80,00 \%)$ & $10(100,00 \%)$ & \\
\hline \multicolumn{5}{|l|}{ Renda Familiar } \\
\hline Até 1 salário mínimo & $4(7,84 \%)$ & $47(92,16 \%)$ & $51(100,00 \%)$ & \multirow{3}{*}{0,288} \\
\hline De 1 a 2 salários mínimos & $5(20,00 \%)$ & $20(80,00 \%)$ & $25(100,00 \%)$ & \\
\hline Mais do que 2 salários mínimos & $0(0,00 \%)$ & $4(100,00 \%)$ & $4(100,00 \%)$ & \\
\hline \multicolumn{5}{|l|}{ Escolaridade } \\
\hline Sem escolaridade & $0(0,00 \%)$ & $5(100,00 \%)$ & $5(100,00 \%)$ & \multirow{3}{*}{0,852} \\
\hline Até 9 anos de estudo & $4(13,79 \%)$ & $25(86,21 \%)$ & $29(100,00 \%)$ & \\
\hline 10 anos de estudo ou mais & $5(10,87 \%)$ & $41(89,13 \%)$ & $46(100,00 \%)$ & \\
\hline
\end{tabular}

Legenda: *Teste qui-quadrado de Pearson

Tabela 4 - Associação do componente Acesso de Primeiro Contato - Utilização com as variáveis sociodemográficas.

\begin{tabular}{|c|c|c|c|c|}
\hline \multirow{3}{*}{ Variáveis } & \multicolumn{4}{|c|}{$\begin{array}{l}\text { Acesso de Primeiro Contato } \\
\text { - Utilização }(\mathbf{N}=\mathbf{8 0})\end{array}$} \\
\hline & $\begin{array}{l}\text { Baixo escore } \\
(<6,6)\end{array}$ & $\begin{array}{l}\text { Alto escore } \\
(\geq \mathbf{6 , 6})\end{array}$ & Total & $\begin{array}{l}\text { Valor } \\
\text { de } p^{*}\end{array}$ \\
\hline & $\mathrm{n}(\%)$ & $\mathrm{n}(\%)$ & $\mathrm{n}(\%)$ & \\
\hline \multicolumn{5}{|l|}{ Sexo } \\
\hline Feminino & $12(18,18 \%)$ & $54(81,82 \%)$ & $66(100,00 \%)$ & \multirow{2}{*}{0,162} \\
\hline Masculino & $5(35,71 \%)$ & $9(64,29 \%$ & $14(100,00 \%)$ & \\
\hline \multicolumn{5}{|l|}{ Idade } \\
\hline Entre 18 e 24 anos & $6(37,50 \%)$ & $10(62,50 \%)$ & $16(100,00 \%)$ & \multirow{3}{*}{$0,036^{\dagger}$} \\
\hline Entre 25 e 59 anos & $10(23,81 \%)$ & $32(76,19 \%)$ & $42(100,00 \%)$ & \\
\hline 60 anos ou mais & $1(4,55 \%)$ & $21(95,45 \%)$ & $22(100,00 \%)$ & \\
\hline \multicolumn{5}{|l|}{ Cor/Raça } \\
\hline Brancos & $3(20,00 \%)$ & $12(80,00 \%)$ & $15(100,00 \%)$ & \multirow{3}{*}{1,000} \\
\hline Pretos e Pardos & $12(21,82 \%)$ & $43(78,18 \%)$ & $55(100,00 \%)$ & \\
\hline Outros & $2(20,00 \%)$ & $8(80,00 \%)$ & $10(100,00 \%)$ & \\
\hline \multicolumn{5}{|l|}{ Renda Familiar } \\
\hline Até 1 salário-mínimo & $11(21,57 \%)$ & $40(78,43 \%)$ & $51(100,00 \%)$ & \multirow{2}{*}{0,811} \\
\hline De 1 a 2 salários-mínimos & $6(24,00 \%)$ & $19(76,00 \%)$ & $25(100,00 \%)$ & \\
\hline
\end{tabular}


Mais do que 2 salários-mínimos

$0(0,00 \%)$

$4(100,00 \%)$

$4(100,00 \%)$

\begin{tabular}{ccccc} 
Escolaridade & & & \\
Sem escolaridade & $0(0,00 \%)$ & $5(100,00 \%)$ & $5(100,00 \%)$ \\
Até 9 anos de estudo & $7(24,14 \%)$ & $22(75,86 \%)$ & $29(100,00 \%)$ & 0,742 \\
\cline { 1 - 2 } 10 anos de estudo ou mais & $10(21,74 \%)$ & $36(78,26 \%)$ & $46(100,00 \%)$ &
\end{tabular}

Legenda: *Teste qui-quadrado de Pearson; ${ }^{\dagger}$ Valor de $\mathrm{p}<0,05$.

Tabela 5 - Associação do componente Acesso de Primeiro Contato - Acessibilidade com as variáveis sociodemográficas.

\begin{tabular}{|c|c|c|c|c|}
\hline \multirow{3}{*}{ Variáveis } & \multicolumn{4}{|c|}{$\begin{array}{l}\text { Acesso de Primeiro Contato } \\
\text { - Acessibilidade }(\mathbf{N}=\mathbf{8 0})\end{array}$} \\
\hline & $\begin{array}{c}\text { Baixo escore } \\
(<6,6)\end{array}$ & $\begin{array}{l}\text { Alto escore } \\
\quad(\geq \mathbf{6 , 6})\end{array}$ & Total & $\begin{array}{l}\text { Valor } \\
\text { de p* }\end{array}$ \\
\hline & $\mathrm{n}(\%)$ & $\mathrm{n}(\%)$ & $\mathrm{n}(\%)$ & \\
\hline \multicolumn{5}{|l|}{ Sexo } \\
\hline Feminino & $59(89,39 \%)$ & $7(10,61 \%)$ & $66(100,00 \%)$ & \multirow{2}{*}{0,095} \\
\hline Masculino & $10(71,43 \%)$ & $4(28,57 \%)$ & $14(100,00 \%)$ & \\
\hline \multicolumn{5}{|l|}{ Idade } \\
\hline Entre 18 e 24 anos & $15(93,75 \%)$ & $1(6,25 \%)$ & $16(100,00 \%)$ & \multirow{3}{*}{0,423} \\
\hline Entre 25 e 59 anos & $37(88,10 \%)$ & $5(11,90 \%)$ & $42(100,00 \%)$ & \\
\hline 60 anos ou mais & $17(77,27 \%)$ & $5(22,73 \%)$ & $22(100,00 \%)$ & \\
\hline \multicolumn{5}{|l|}{ Cor/Raça } \\
\hline Brancos & $13(86,67 \%)$ & $2(13,33 \%)$ & $15(100,00 \%)$ & \multirow{3}{*}{1,000} \\
\hline Pretos e Pardos & $37(67,27 \%)$ & $18(32,73 \%)$ & $55(100,00 \%)$ & \\
\hline Outros & $8(80,00 \%)$ & $2(20,00 \%)$ & $10(100,00 \%)$ & \\
\hline \multicolumn{5}{|l|}{ Renda Familiar } \\
\hline Até 1 salário mínimo & $46(90,20 \%)$ & $5(9,80 \%)$ & $51(100,00 \%)$ & \multirow{3}{*}{0,280} \\
\hline De 1 a 2 salários mínimos & $20(80,00 \%)$ & $5(20,00 \%)$ & $25(100,00 \%)$ & \\
\hline Mais do que 2 salários mínimos & $3(75,00 \%)$ & $1(25,00 \%)$ & $4(100,00 \%)$ & \\
\hline \multicolumn{5}{|l|}{ Escolaridade } \\
\hline Sem escolaridade & $4(80,00 \%)$ & $1(20,00 \%)$ & $5(100,00 \%)$ & \multirow{3}{*}{0,482} \\
\hline Até 9 anos de estudo & $24(82,76 \%)$ & $5(17,24 \%)$ & $29(100,00 \%)$ & \\
\hline 10 anos de estudo ou mais & $41(89,13 \%)$ & $5(10,87 \%)$ & $46(100,00 \%)$ & \\
\hline
\end{tabular}

Legenda: *Teste qui-quadrado de Pearson

Tabela 6 - Associação do componente Coordenação - Integração do Cuidado com as variáveis sociodemográficas.

\begin{tabular}{cccccc} 
& \multicolumn{5}{c}{ Coordenação - Integração do cuidado $(\mathbf{N}=\mathbf{8 0})$} \\
Variáveis & $\begin{array}{c}\text { Baixo escore } \\
(<\mathbf{6 , 6})\end{array}$ & $\begin{array}{c}\text { Alto escore } \\
(\geq 6,6)\end{array}$ & $\begin{array}{c}\text { Não } \\
\text { avaliado }\end{array}$ & Total & $\begin{array}{c}\text { Valor } \\
\text { de } \mathbf{p}^{*}\end{array}$ \\
\cline { 2 - 5 } & $\mathrm{n}(\%)$ & $\mathrm{n}(\%)$ & $\mathrm{n}(\%)$ & $\mathrm{n}(\%)$ &
\end{tabular}




\begin{tabular}{|c|c|c|c|c|c|}
\hline Sexo & & & & & \multirow{3}{*}{0,106} \\
\hline Feminino & $19(28,79 \%)$ & $33(50,00 \%)$ & $14(21,21 \%)$ & $66(100,00 \%)$ & \\
\hline Masculino & $5(35,71 \%)$ & $3(21,43 \%)$ & $6(42,86 \%)$ & $14(100,00 \%)$ & \\
\hline \multicolumn{6}{|l|}{ Idade } \\
\hline Entre 18 e 24 anos & $7(43,75 \%)$ & $5(31,25 \%)$ & $4(25,00 \%)$ & $16(100,00 \%)$ & \multirow{3}{*}{0,476} \\
\hline Entre 25 e 59 anos & $12(28,57 \%)$ & $18(42,86 \%)$ & $12(28,57 \%)$ & $42(100,00 \%)$ & \\
\hline 60 anos ou mais & $5(22,73 \%)$ & $13(59,09 \%)$ & $4(18,18 \%)$ & $22(100,00 \%)$ & \\
\hline \multicolumn{6}{|l|}{ Cor/Raça } \\
\hline Brancos & $5(33,33 \%)$ & $7(46,67 \%)$ & $3(20,00 \%)$ & $15(100,00 \%)$ & \multirow{3}{*}{0,983} \\
\hline Pretos e Pardos & $16(29,09 \%)$ & $25(45,46 \%)$ & $14(25,45 \%)$ & $55(100,00 \%)$ & \\
\hline Outros & $3(30,00 \%)$ & $4(40,00 \%)$ & $3(30,00 \%)$ & $10(100,00 \%)$ & \\
\hline \multicolumn{6}{|l|}{ Renda Familiar } \\
\hline Até 1 salário-mínimo & $14(27,45 \%)$ & $26(50,98 \%)$ & $11(21,57 \%)$ & $51(100,00 \%)$ & \multirow{3}{*}{0,575} \\
\hline $\begin{array}{l}\text { De } 1 \text { a } 2 \\
\text { salários-mínimos }\end{array}$ & $8(32,00 \%)$ & $9(36,00 \%)$ & $8(32,00 \%)$ & $25(100,00 \%)$ & \\
\hline $\begin{array}{l}\text { Mais do que } 2 \text { salários- } \\
\text { mínimos }\end{array}$ & $2(50,00 \%)$ & $1(25,00 \%)$ & $1(25,00 \%)$ & $4(100,00 \%)$ & \\
\hline \multicolumn{6}{|l|}{ Escolaridade } \\
\hline Sem escolaridade & $1(20,00 \%)$ & $4(80,00 \%)$ & $0(0,00 \%)$ & $5(100,00 \%)$ & \multirow{3}{*}{0,501} \\
\hline Até 9 anos de estudo & $7(24,14 \%)$ & $14(48,28 \%)$ & $8(27,59 \%)$ & $29(100,00 \%)$ & \\
\hline $\begin{array}{c}10 \text { anos de estudo } \\
\text { ou mais }\end{array}$ & $16(34,78 \%)$ & $18(39,13 \%)$ & $12(26,09 \%)$ & $46(100,00 \%)$ & \\
\hline
\end{tabular}

Legenda: *Teste qui-quadrado de Pearson

\section{Discussão}

A incorporação da opinião do usuário na avaliação dos serviços de saúde tem sido valorizada e relacionada à melhora na adesão ao tratamento e a um maior vínculo entre o serviço de saúde e o usuário. Nas últimas décadas, diversos estudos, nacionais $^{11,17,18}$ e internacionais ${ }^{19,20}$, têm incluído o ponto de vista dos usuários nos processos avaliativos, tendo uma significativa importância na contribuição para os estudos em APS, uma vez que permite intervenções mais adequadas para solucionar problemas presentes no cotidiano dos serviços, propiciando avanços no âmbito da produção de cuidados e gestão dos serviços de saúde.

Quando comparado ao cenário internacional $^{21,22}$, o Brasil ainda apresenta escassez de trabalhos que avaliam os serviços de saúde na perspectiva de seus usuários. Entretanto, fica claro que o desafio atual é tornar a avaliação em saúde sob a ótica dos usuários uma prática institucionalizada de modo a considerá-la como reordenadora e parte integral das operações diárias da gestão em saúde.

Em se tratando das dimensões avaliadas, o grau de afiliação visa identificar o serviço que serve como referência para o usuário, funcionando como fonte regular de atenção à sua saúde. Os resultados deste estudo mostraram que essa dimensão obteve o maior escore $(8,9)$ entre todos os componentes avaliados. Esse resultado é semelhante ao encontrado por Duarte et al. ${ }^{23}$ que avaliou a opinião dos usuários atendidos nas UBS de um município da região centro-oeste de Minas 
Gerais e observou que o grau de afiliação foi uma das dimensões mais bem avaliadas no estudo. A avaliação positiva dessa dimensão reflete a importância da equipe de saúde da família para os usuários que, muitas vezes, possuem apenas na APS o referencial de cuidado à saúde, ratificando o que é preconizado nas bases organizativas da $\mathrm{PNAB}^{3}$, quanto à capacidade de vinculação e responsabilização do cuidado por parte das ESF, sendo fundamentais para a efetivação da atenção básica como primeiro contato da rede de atenção à saúde.

No que diz respeito a cada um dos atributos da APS, a garantia do acesso de primeiro contato, em suas dimensões de utilização e acessibilidade, está relacionada com o uso da APS como a porta de entrada aberta e preferencial da rede de atenção, sendo fonte de cuidado a cada novo problema de saúde, com exceção das verdadeiras emergências e urgências médicas. Esse atributo é constituído pela dimensão utilização que diz respeito aos processos estabelecidos no atendimento aos usuários e pela acessibilidade a qual remete à estrutura disponível.

O componente utilização obteve um alto escore $(7,7)$, em semelhança com o estudo de Araújo et al. ${ }^{24}$ que avaliou a perspectiva da qualidade do cuidado na APS em pessoas idosas e teve a utilização como a melhor dimensão avaliada entre todos os atributos. Esse resultado demonstra que o processo de trabalho das equipes em relação ao primeiro contato é muito bem avaliado, evidenciando a presença de uma inter-relação entre o serviço de APS e o receptor do cuidado.

Em relação à dimensão acessibilidade, obteve-se escore abaixo do desejado $(3,3)$. Diversas pesquisas já realizadas evidenciaram que é a pior dimensão avaliada ${ }^{17,25}$, corroborando com os resultados do presente estudo. Os usuários percebem o acesso ao serviço da APS como algo burocrático e demorado, o que representa a não valorização da ESF como locus capaz de resolver problemas de saúde da população. Portanto, os usuários tendem a buscar outras formas de conseguir atendimento a demandas que poderiam ser solucionadas na APS, sobrecarregando, assim, outros serviços e comprometendo o alcance pleno do atributo acesso de primeiro contato da APS. ${ }^{15}$

O componente coordenação integração de cuidado é considerado pilar da concepção estruturante e complexa da APS, em que pressupõe alguma forma de continuidade do cuidado, seja por meio de acompanhamento pelo mesmo profissional e/ou pelos prontuários médicos, seja pelo reconhecimento dos problemas abordados em outros serviços ${ }^{6}$. Essa dimensão também recebeu avaliação positiva na percepção dos usuários $(6,8)$. O resultado está em consonância com a pesquisa de Araújo et $a l .{ }^{25}$ que foi realizada nas UBS de um município do Paraná e identificou um alto escore da dimensão coordenação integração do cuidado sob a perspectiva dos cuidadores de crianças menores de 12 anos. Por sua vez, a avaliação positiva desse atributo indica a presença de articulação entre os diversos níveis assistenciais. No entanto, apesar de ser considerado bem avaliado, o resultado 6,8 está próximo ao ponto de corte considerado para baixo escore $(<6,6)$, isso mostra que há uma fragilização dos processos de coordenação e continuidade do cuidado ao longo da rede de atenção à saúde.

$\mathrm{Na}$ avaliação das dimensões da APS quando analisados comparativamente em relação aos diferentes fatores sociodemográficos, não se verificou diferença estatisticamente significante, com exceção do componente acesso de primeiro contato - utilização em relação à faixa etária. Percebeu-se que, em valores percentuais, a avaliação positiva do item utilização foi maior entre os idosos com diferença estatisticamente significativa $(p<0,05)$, o que revela uma melhor avaliação desse atributo pelos idosos. Alves et al. ${ }^{14}$ encontraram resultados semelhantes quando avaliaram usuários do sexo masculino em um município da Paraíba. Esse resultado pode estar relacionado a uma 
maior utilização dos usuários pelo serviço de saúde em função da idade, fato que pode ser explicado pelas doenças crônicas decorrentes do envelhecimento, necessitando de acompanhamento a longo prazo.

$\mathrm{Na}$ presente pesquisa, observou-se uma predominância do sexo feminino no perfil dos usuários, em que há uma concordância com diversos estudos realizados tanto nacionais ${ }^{13,26,27}$ quanto internacionais ${ }^{19}$. Isso remete a uma situação social em que a mulher assume papel nuclear na família em relação aos cuidados com a saúde, acessando com mais frequência os serviços de saúde, em comparação ao sexo masculino. Embora os homens possuam maiores taxas de morbimortalidade, eles resistem mais a buscar assistência à saúde, dificultando a avaliação da percepção masculina para os serviços de saúde em geral.

Embora a amostra desse trabalho tenha representação de usuários dos oito distritos sanitários do Recife-PE, deve-se considerar como limitação a representabilidade quanto ao número de USF e, consequentemente, da amostra, tendo assim um caráter mais exploratório por abarcar 8 das 132 USF da cidade do Recife. Todavia, apesar da limitação indicada, ficou evidente a importância da APS para os usuários, visto que reconhecem de maneira satisfatória componentes essenciais como o grau de afiliação, o acesso de primeiro contato - utilização e a coordenação - integração do cuidado. Além disso, identificou-se que ainda há muitas barreiras a serem enfrentadas com relação à saúde, mas uma das mais importantes a serem vencidas é a do acesso. Portanto, sugere-se, ainda, que sejam realizados estudos que visem subsidiar conhecimento com a finalidade de promover um melhor acesso à atenção primária, sobretudo com vistas à garantia da acessibilidade estruturalmente.

\section{Conclusão}

Os escores evidenciados pelo instrumento de avaliação PCATool-Brasil demonstraram que os usuários da APS no município do Recife - PE consideram a USF como fonte contínua de atenção às suas necessidades de saúde ao longo da vida. Porém, ficou claro que os usuários encontram dificuldades na acessibilidade ao serviço de saúde. Portanto, apesar da APS ser a porta de entrada preferencial para o sistema de saúde, percebeu-se a necessidade de mudanças no sentido de tornar o serviço menos burocrático.

Constatou-se, também, que a posição da USF como centro coordenador do cuidado nos demais níveis assistenciais é reconhecida. No entanto, a avaliação indica que esse atributo ainda necessita de aperfeiçoamentos. Além disso, observou-se que a faixa etária mais idosa apresenta melhores avaliações no quesito da utilização do acesso de primeiro contato com a USF, sendo um ponto positivo já que muitos idosos necessitam de acompanhamento a longo prazo por causa de doenças crônicas decorrentes da idade.

Espera-se que os resultados dessa pesquisa permitam subsidiar a reformulação e execução de ações de melhoramento no âmbito da política de saúde municipal e nacional, bem como do fortalecimento da avaliação em saúde na ESF pela perspectiva do usuário.

\section{Referências}

1. OPAS/OMS. Declaração-Alma-Ata. In: Conferência Internacional sobre Cuidados Primários de Saúde [Internet]. 1978. Available from: http://www.opas.org.br

2. Gusso G, Lopes JMC. Tratado de Medicina de Família e Comunidade. 2 ed. Artmed, editor. Porto Alegre; 2012.

3. Brasil M da S. Política nacional da atenção basica. 2012. 
4. Costa EC, Cristina F, Pinto DL, Israel P, Lira C De. Percepções e práticas profissionais no cuidado da obesidade na estratégia saúde da família. 2020;18:85-100.

5. Starfield B. Atenção Primária: equilíbrio entre necessidades de saúde, serviços e tecnologia. UNESCO, Ministério da Saúde. Brasília; 2002. 726 p.

6. Brasil $\mathrm{M}$ da $\mathrm{S}$. Manual do Instrumento de Avaliação da Atenção Primária à Saúde. Ministério. Brasíllia DF, editor. Brasilia; 2010. 82 p.

7. Macinko J, Mendonça CS. Estratégia Saúde da Família, um forte modelo de Atenção Primária à Saúde que traz resultados. Saúde em Debate. 2018;42(spe1):18-37.

8. Araújo KMDFA, Lana FCF. Relação da Hanseníase com a cobertura da Estratégia Saúde da Família e condições socioeconômicas. Cienc y Enferm. 2020;26.

9. Brentani A, Grisi SJFE, Taniguchi MT, Ferrer APS, de Moraes Bourroul ML, Fink G. Rollout of community-based family health strategy (programa de saude de familia) is associated with large reductions in neonatal mortality in São Paulo, Brazil. SSM - Popul Heal. 2016;2:55-61.

10. Sousa MF de, Hamann EM. Programa Saúde da Família no Brasil: uma agenda incompleta? Cien Saude Colet. 2009;14(Suppl 1):1325-35.

11. Zils ADA, Castro RCL de, Oliveira MMC de, Harzheim E, Duncan BB. Satisfação dos usuários da rede de Atenção Primária de Porto Alegre. Rev Bras Med Família e Comunidade. 2016;4(16):270-6.

12. Donabedian A. La calidad de la atención medica: definición, método e evaluación. Mexicana LPM, editor. México; 1984.

13. Paula W, Silva S, Samico IC, Filho MB, Caminha M de F. Avaliação da atenção básica à saúde sob a ótica dos usuários: uma revisão sistemática. Rev Esc Enferm USP. 2016;50(2):335-45.

14. Alves A do N, Coura AS, França ISX de, Magalhães IM de O, Rocha MA, Araújo R da S. Acesso de primeiro contato na atenção primária: uma avaliação pela população masculina. Rev Bras Epidemiol. 2020;23:e200072.

15. Saúde SM de. Plano Municipal de Saúde da Prefeitura da Cidade do Recife 2018-2021. 2018;95.

16. Harzheim E, Oliveira MMC de, Agostinho MR, Hauser L, Stein AT, Gonçalves MR, et al. Validação do instrumento de avaliação da atenção primária à saúde: PCATool-Brasil adultos. Rev Bras Med Família e Comunidade. 2013;8(29):274-84.

17. Gontijo TL, Duarte AGS, Guimarães EA de A, Silva J da. Avaliação da atenção primária: o ponto de vista de usuários. Saúde em Debate. 2017;41(114):741-52.

18. Furtado MC de C, Braz JC, Pina JC, de Mello DF, de Lima RAG. A avaliação da atenção à saúde de crianças com menos de um ano de idade na Atenção Primária. Rev Lat Am Enfermagem [Internet]. 2013;21(2):554-61.

19. Wei X, Li H, Yang N, Wong SYS, Owolabi O, Xu J, et al. Comparing quality of public primary care between Hong Kong and Shanghai using validated patient assessment tools. PLoS One. 2015;10(3):1-15.

20. Berra S, Rodríguez-Sanz M, Rajmil L, Pasarín MI, Borrell C. Experiences with primary care associated to health, socio-demographics and use of services in children and adolescents. Cad Saude Publica. 2014;30(12):2607-18.

21. Dantas CR, Oda AMGR. Cartografia das pesquisas avaliativas de serviços de saúde mental no Brasil (2004-2013). Vol. 24, Physis. 2014. 1121-1279 p.

22. Trapé TL, Campos RTO, Costa KS. Rede de Atenção à Saúde Mental: estudo comparado Brasil e Catalunha. Physis Rev Saúde Coletiva. 2018;28(4):1-19.

23. Duarte AGS, Gontijo TL, Guimarães EA de A, Cavalcante RB, Belo VS, Silva GS. Fatores associados ao desempenho de serviços da atenção primária à saúde. Rev Bras em Promçoção da Saúde. 2019;32:1-10. 
24. Araújo LUA de, Gama ZA da S, Nascimento FLA do, Oliveira HFV de, Azevedo WM de, Almeida Júnior HJB de. Avaliação da qualidade da atenção primária à saúde sob a perspectiva do idoso. Cien Saude Colet. 2014;19(8):3521-32.

25. Araujo JP, Viera CS, Oliveira BRG, Gaiva MA, Rodrigues RM. Avaliação dos atributos essenciais da Atenção Primária à Saúde da criança. Rev Bras Enferm [Internet]. 2018;71(suppl 3):1447-54.

26. Guibu IA, de Moraes JC, Junior AAG, Costa EA, Acurcio F de A, Costa KS, et al. Características principais dos usuários dos serviços de atenção primária à saúde no Brasil. Rev Saude Publica. 2017;51(Supl 2:17s):1s-13s.

27. Bandeira D, Damaceno AN, Weiller TH, Lopes LFD. Evaluation of the Coordination of Care By Users of Primary Health Care Services. Reme Rev Min Enferm. 2020;24:1-9.

\section{Como citar este artigo:}

Freitas CGM, Silva TCL, Gomes NPCP. Avaliação da rede de atenção primária do município de Recife - PE sob a ótica de um grupo de usuários. Rev. Aten. Saúde. 2021; 19(67): 314- 
Freitas CGM, Silva TCL, Gomes NPCP

328. 